\title{
Label-Free Imaging and Optical Characterization of Tissues Based on Autofluorescence
}

\author{
Sourav Bhattacharjee, ${ }^{*}{ }^{\dagger}$ Cimran Satwaha, ${ }^{\ddagger}$ Kevin Thornton, ${ }^{\dagger}$ and Dimitri Scholz ${ }^{\S}$ \\ ${ }^{\dagger}$ School of Veterinary Medicine and ${ }^{\S}$ Conway Institute of Biomolecular and Biomedical Research, University College Dublin (UCD), \\ Belfield, Dublin 4, Ireland \\ ${ }^{\ddagger}$ School of Life Sciences, Pharmacy and Chemistry, Kingston University, London, U.K.
}

Supporting Information

ABSTRACT: Attaining capability of label-free optical characterization of tissues will offer methodological advancement and possibilities for early clinical detection, which is of paramount importance in treating patients under clinical setups, for example, cancer. Here, we demonstrate the potential of autofluorescence exhibited by tissues as an enabling microscopic strategy to achieve high-resolution imagery data offering a wealth of clinically relevant information including possibility

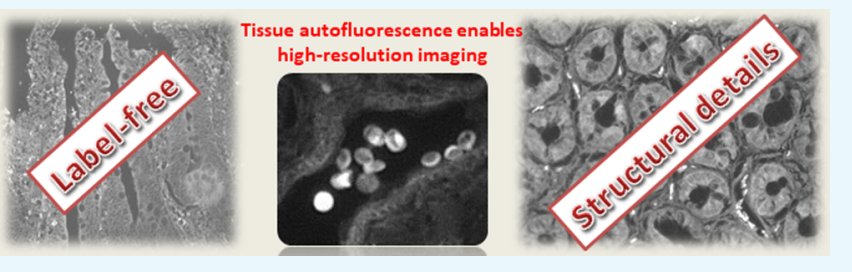
of three-dimensional rendering. Furthermore, we elucidate the use of analytic tools to extract numerical read-outs from such data with further implications in histopathology, pharmaceutics, toxicology, and screening purposes. This study summarizes the results obtained through a systematic autofluorescence-based investigation on murine and porcine gut tissues with an example of applying the technique in nanotoxicology. The study provides with a methodological roadmap toward developing a fast, effective, and robust platform enabling in-depth optical characterization of tissues.

\section{INTRODUCTION}

Acquiring high-resolution images from tissue samples in light microscopy remains a challenge. The opaque, dense, and granular matrix in tissue blocks causes scattering of light and prevents adequate penetration resulting in loss of useful structural, pathological, and clinically relevant information. ${ }^{1}$ With significant advancement in fluorescence microscopy, plenty of fluorophores, including immunolabeling tools, have been developed offering opportunities to tag and image various cellular and subcellular structures. However, as reactive species, fluorophores induce physicochemical and molecular alterations within tissues. Labeling protocols are often time-consuming and require extensive tissue manipulation which is deleterious to native tissue structure. ${ }^{2}$ Moreover, commercialized labeling kits are expensive and require infrastructural support. As a result, conventional hematoxylin and eosin $(\mathrm{H} \& \mathrm{E})$-stained histopathology slides continue to be popular in clinical diagnostics, despite extensive tissue manipulation and paucity of data due to lack of three-dimensional (3D) representation. Switching to label-free fluorescence microscopy will thus offer an edge over existing labeling-derived or H\&E staining protocols on the merits of being less laborious, cost-effective, minimal tissue manipulation, and, above all, providing 3D information. Autofluorescence from tissues, as exemplified here, can be an interesting strategy. It is known that various tissues (e.g., blood), tissue components (e.g., elastin, collagen), or intracellular molecules (e.g., flavins, reduced nicotinamide adenine dinucleotide phosphate, amino acids such as tyrosine, tryptophan, and phenylalanine) act as endogenous fluorophores. $^{3-9}$ Porphyrin, a digested blood-borne product present in processed tissues, is particularly reported to exhibit excellent emission, ${ }^{10}$ which makes it an exciting tool to image tissues, especially the ones rich in blood supply, for example, the gut. This report provides further in-depth analysis on the utility of tissue autofluorescence toward designing an effective tissue characterization and histological platform. Although autofluorescence has been used before to image isolated cells (e.g., leukocytes), ${ }^{11}$ its use in characterizing tissue is scarce.

\section{RESULTS AND DISCUSSION}

Porcine and murine jejunal tissue slides were prepared without any staining by the standard protocol (Figure S1) and then visualized in an inverted microscope. Acquisition under an identical setup (e.g., intensity, exposure time, image gain, aperture size, etc.) provided comparability. Images with excellent resolution were acquired from the tissue slides under blue $\left(\lambda_{\text {ex } / \mathrm{em}} 470 / 525 \mathrm{~nm}\right)$ channel (Figure 1a) and reasonable signal was obtained under $\operatorname{UV}\left(\lambda_{\mathrm{ex} / \mathrm{em}} 357 / 445 \mathrm{~nm}\right)$, whereas no signal was received in green $\left(\lambda_{\mathrm{ex} / \mathrm{em}} 560 / 630 \mathrm{~nm}\right)$ or red $\left(\lambda_{\text {ex/em }} 628 / 692 \mathrm{~nm}\right)$ channels. The overall texture of both porcine and murine jejunal tissues was visible with high definition. Crisp quality images enabled further zooming into specific substructures [e.g., villi, red blood corpuscle (RBC), smooth muscles, and glands] delving deeper into details such as lamina propria of villi (Figure $1 \mathrm{~b}$ ). The RBCs were visible as biconcave discs and expectedly demonstrated intense emission

Received: April 8, 2018

Accepted: May 23, 2018

Published: May 31, 2018 

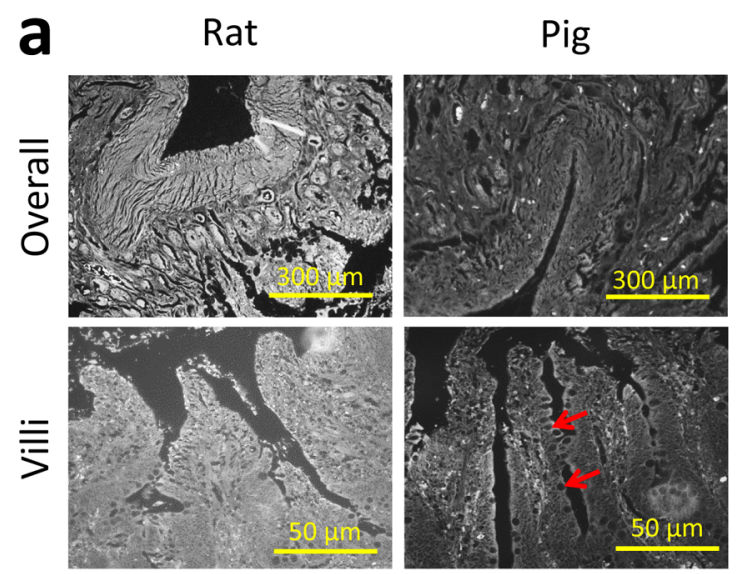

b
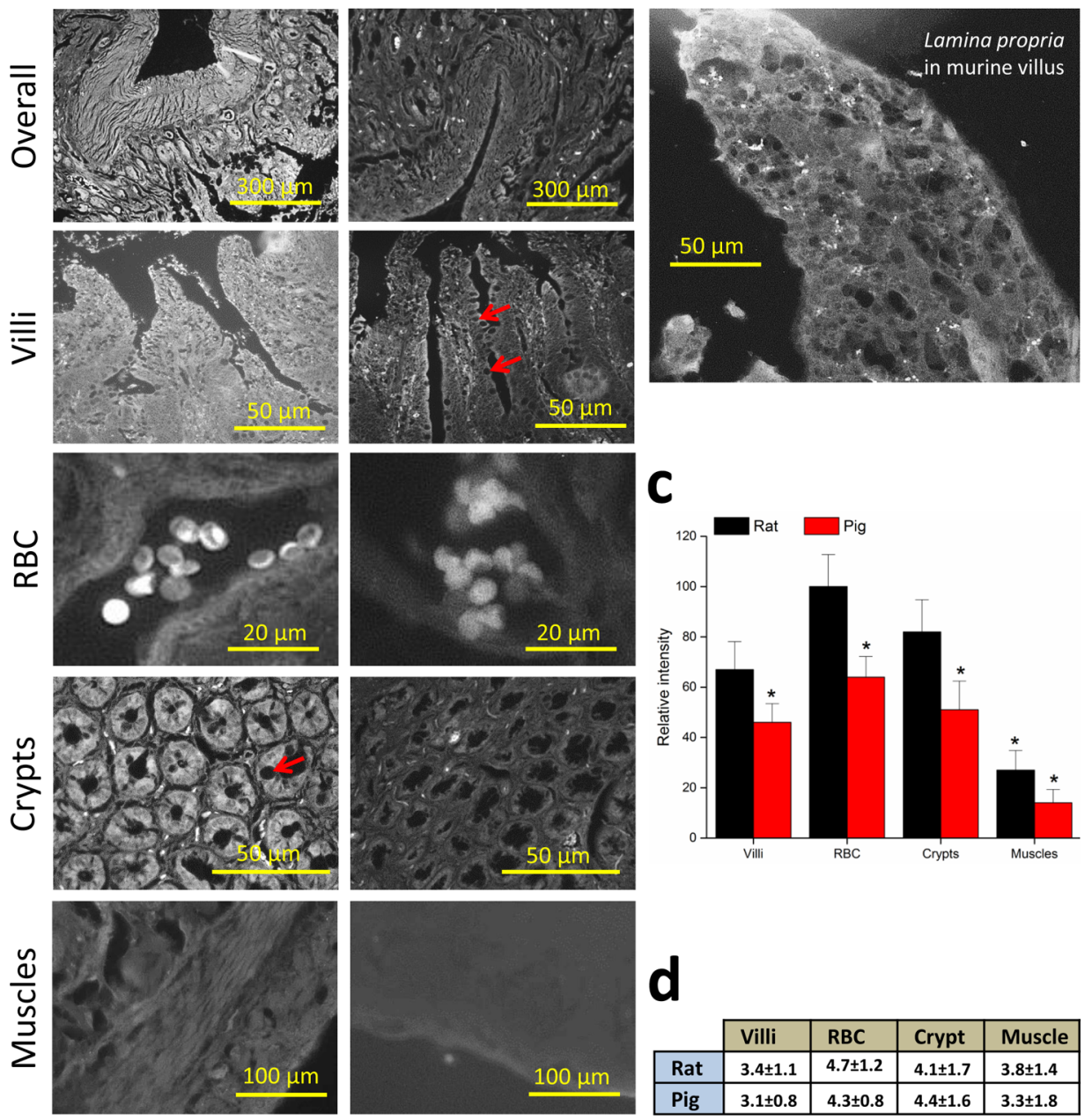

Figure 1. High-resolution imaging and optical characterization of various tissue structures based on autofluorescence in blue $\left(\lambda_{\mathrm{ex}}=470 \mathrm{~nm} ; \lambda_{\mathrm{em}}=\right.$ $525 \mathrm{~nm}$ ) channel. Scale bars are provided as insets. (a) Panel illustrating acquired crisp quality images of overall tissue architecture, villi, RBCs, crypts, and muscles from both murine and porcine jejunum. The red arrows indicate goblet cells. (b) Image of murine villus showing detailed trabeculae of lamina propria. (c) Comparison of emission from different tissue components from both murine and porcine samples. The murine RBCs showed the highest emission (measured in arbitrary units) and all other measurements were expressed as \% to it. Significantly different points $(p<0.05)$ compared to murine RBCs are marked with an asterisk (*). (d) Fluorescence lifetime $/ \tau$ (ns) of various tissue structures in murine and porcine samples measured by FLIM $(n=20)$. All data are presented as mean \pm standard deviation $(\mathrm{SD})(n=20)$. The sharpness of porcine images was adjusted for clarity.

because of elevated levels of porphyrin due to high hemoglobin content. The muscles showed lesser emission from structural proteins (elastin, collagen). The intestinal crypts of Lieberkühn were clearly visible and were studded with mucus-secreting goblet cells. 3D rendering of intestinal villi from $z$-stacks (Video S2) enabled understanding of its internal fabric. Comparison of images revealed higher autofluorescence from murine samples to porcine ones (Figure 1c) because of species variation of hemoglobin and difference in tissue composition. The fluorescence lifetime $(\tau)$ of muscles, glands, and RBCs was assessed by fluorescence lifetime imaging microscopy (FLIM) (Figure 1d).

Characterization of the gut tissue based on autofluorescence offered opportunity to extract numerical read-outs enabling toxicity investigation. Here, we demonstrated an example of exposure scenario to CdSe quantum dots (QDs) $\left(\lambda_{\mathrm{em}}=620 \pm\right.$ $5 \mathrm{~nm}$ in red channel) which are known to cause toxicity. ${ }^{12-16}$ Slices of murine jejunal tissue $(\sim 1 \mathrm{~cm}$ thickness) were placed on a glass slide forming small wells with the gut lumen facing upward while $5 \mu \mathrm{L}$ aliquots of QD-suspension $(100 \mu \mathrm{g} / \mathrm{mL})$ in $\mathrm{N}$-(2-hydroxyethyl)piperazine- $\mathrm{N}^{\prime}$-ethanesulfonic acid (HEPES) buffer were deposited in the lumen (Figure 2a) to allow permeation. After an hour, tissue slides were prepared from exposed slices and imaged in blue (detects tissue autofluorescence and also QDs due to its broad excitation spectrumFigure S3) and red channels (detects only QDs as the tissue did not show autofluorescence in this channel). The structural changes in villi due to the exposure could be identified and the QDs seemed to cluster inside the tissue (Figure 2b) while also penetrated the intestinal crypts (Figure 2c). Numerical data on intratissue distribution of QDs based on the distance from the exposure site (luminal wall) could be developed from a wider (4X) field (Figure 2d). This strategy can be used for quantification (from area under curve) in transport studies with relevance in pharmaceutics. The trabeculae of lamina propria from control and exposed jejunal villi were compared after analysis by BoneJ plug-in based on the mean branch length (Figure 2e,f), connectivity density (Figure 2g), 

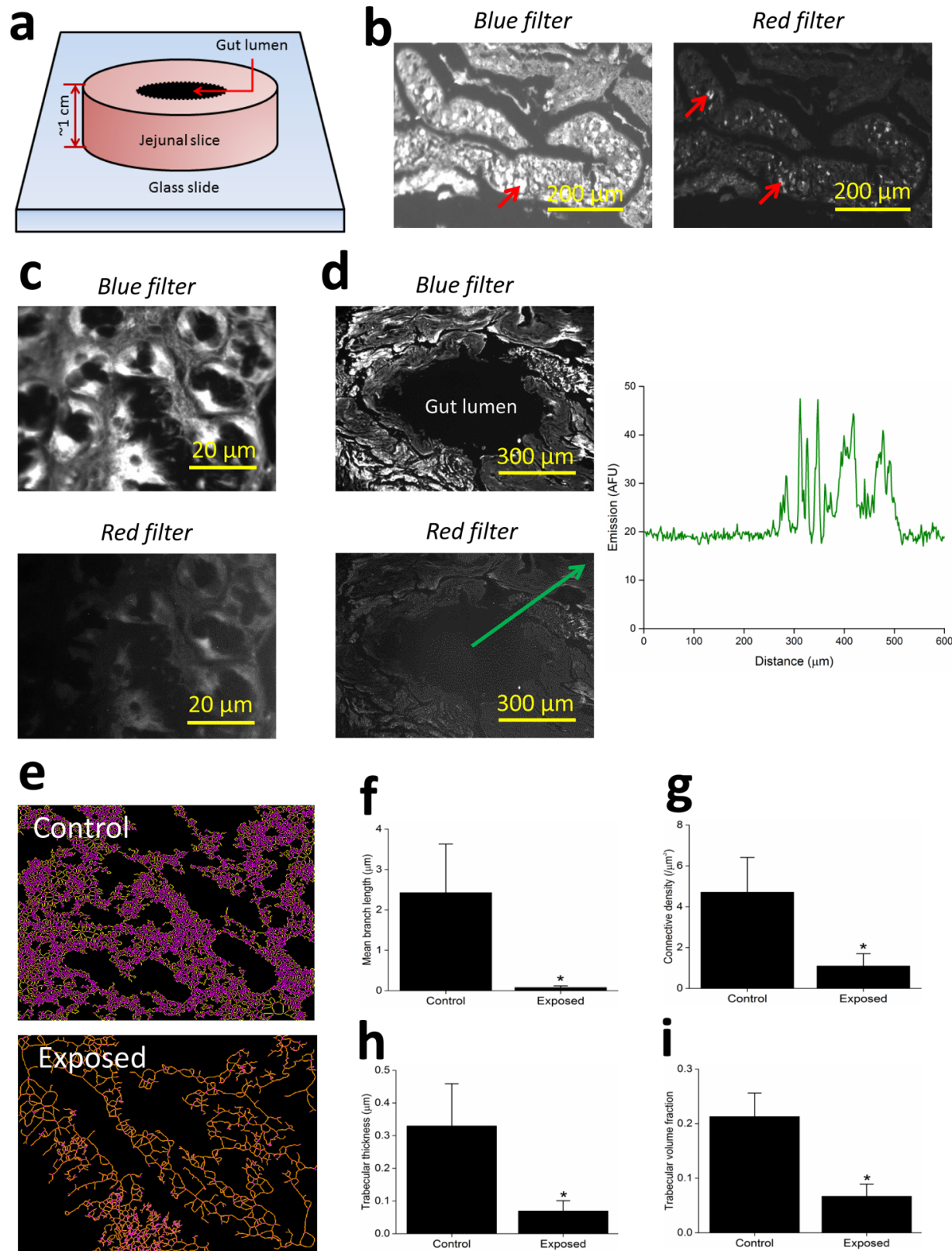

Figure 2. Evaluation of CdSe QD exposure and toxicity caused to murine jejunal tissue. Scale bars are provided as insets. (a) Cartoon showing the exposure setup, where $\sim 1 \mathrm{~cm}$ thick murine jejunal slices were placed over a glass slide creating a well. Around $5 \mu \mathrm{L}$ of aliquots of CdSe dispersion $(100 \mu \mathrm{g} / \mathrm{mL})$ was deposited into the lumen to induce toxicity. High-resolution imaging on (b) jejunal tissue and (c) intestinal crypts under blue $\left(\lambda_{\mathrm{ex} / \mathrm{em}} 470 / 525 \mathrm{~nm}\right)$ and red $\left(\lambda_{\mathrm{ex} / \mathrm{em}} 628 / 692 \mathrm{~nm}\right)$ channels. The blue channel showed both tissue autofluorescence and QDs because of the wide absorbance spectra of QDs. The red channel showed only the QDs inside tissues as no tissue autofluorescence was detected in the red channel. The red arrows point toward clusters of QDs inside tissue. (d) Plot profiling of distribution pattern of QDs inside tissue following the green arrow. This technique can be useful for quantification during transport studies. (e) Trabecular analysis of villous lamina propria with BoneJ plug-in of FIJI and expressed as color-coded image. The control tissue showed higher branching as depicted by accentuation of purple-colored threads, whereas the branching decreased considerably in exposed tissue. $(\mathrm{f}-\mathrm{i})$ Comparing the control and exposed tissue based on numerical read-outs on various parameters (mean branch length, connective density, trabecular thickness, and trabecular volume fraction) to assess toxicity due to CdSe QDs. All data are shown as mean $\pm \mathrm{SD}(n=20)$. Exposed data points significantly different $(p<0.05)$ to control were marked with an asterisk $(*)$.

trabecular thickness (Figure $2 \mathrm{~h}$ ), trabecular volume fraction, and surface area (Figure 2i and Videos S4 + S5). Exposure to QDs resulted in significant decrease in the mean branch length, connectivity density, trabecular thickness, and volume fraction of tissue mass within trabecular network of lamina propria.

\section{CONCLUSIONS}

Although autofluorescence is often cited as the source of interference or background noise in experimental data, it also offers unprecedented opportunities for high-resolution labelfree imaging of tissue with projected applications in transport studies, histopathology on biopsy material, drug development, toxicology, and screening. Furthermore, the presence of such autofluorescence is not limited to the gut and also observed in other tissues. The endogenous fluorophores are highly sensitive molecules capable of detecting minute chemical changes in their surroundings. For example, fluorescence lifetime $(\tau)$ is a highly sensitive concentration-independent parameter ${ }^{17}$ and 
thus can be used to spot pathology in the gut, for example, neoplastic (cancerous lesions, ulceration, and polyps), inflammatory (colitis), hemoglobinopathies, or structural defects in RBCs at early stages. Tissue handling is crucial to achieve highquality, consistent, and comparable data. Hence, the protocol for preparing tissue slides should be uniform. In this study, structural analysis of villi including trabecular network of lamina propria was performed to assess toxicity. This can be useful for diagnostics in relation to various clinical conditions, for example, villous atrophy found in celiac, ${ }^{18}$ Whipple, ${ }^{19}$ and Crohn's $^{20}$ disease or toxicity from oral exposure. Moreover, advancement in light-emitting diode (LED) technology has now enabled cheap, long-lasting, portable, and energy-efficient light sources compatible with simpler microscopic platforms. Availability of freeware tools and automated microscopic platforms hold additional possibilities for multiplexing, arraybased analysis, microfluidics, and high-content screening.

\section{MATERIALS \& METHODS}

4.1. Quantum Dots. CdSe QDs (6.2-7.7 nm), absorption $620 \mathrm{~nm}$, emission $640 \mathrm{~nm}$ (Lumidot, product no. 662461, Sigma-Aldrich) were purchased $(5 \mathrm{mg} / \mathrm{mL}$ in toluene). Stock dispersions $(1 \mathrm{mg} / \mathrm{mL})$ were made with dilution in ethanol, whereas working dispersions $(100 \mu \mathrm{g} / \mathrm{mL})$ were prepared by further dilution in HEPES buffer (product no. 83264, SigmaAldrich). The excitation and emission spectrums of $100 \mu \mathrm{g} / \mathrm{mL}$ dispersion of the QDs were measured in a spectrofluorometer (Figure S3).

4.2. Jejunal Tissues and Preparation of Tissue Slides. Both murine (male Wistar rats, age 8-10 weeks, wt 250-350 g) and porcine jejunum were acquired from other groups in UCD (refinement of the 3Rs-Replacement, Reduction, and Refinement). The jejunal tissue samples were fixed in $10 \%$ formalin and the paraffin blocks were prepared after processing with repeated cycles of alcoholic dehydration and clearing with xylene. Microtomed tissue sections ( $5 \mu \mathrm{m}$ thickness) were removed from paraffin and mounted on glass slides with thin coverslips using DPX mounting medium.

4.3. Exposure Scenario. Thin cross-sectional slices $(\sim 1$ $\mathrm{cm}$ thickness) from murine jejunum were dissected and deposited on a glass slide with creation of a well, where circular tissue formed the rim and lumen faced upward (Figure 2a). Five microliter aliquots of QD-dispersion $(100 \mu \mathrm{g} / \mathrm{mL})$ were then deposited in the lumen and left for an hour, followed by thorough washing of the tissue in water and preparation of slides as described above.

4.4. Microscopy. Tissue slides were examined ( $4 \times, 10 \times$, $20 \times, 40 \times)$ in an inverted Zeiss AxioVision microscope with LED light source under four channels: UV $\left(\lambda_{\mathrm{ex} / \mathrm{em}} 357 / 445\right.$ $\mathrm{nm})$, blue $\left(\lambda_{\text {ex/em }} 470 / 525 \mathrm{~nm}\right)$, green $\left(\lambda_{\text {ex/em }} 560 / 630 \mathrm{~nm}\right)$, and red $\left(\lambda_{\text {ex } / \mathrm{em}} 628 / 692 \mathrm{~nm}\right)$. Images were stored as .zvi files for further analysis. FLIM was performed with a $10 \times$ objective in an inverted LIFA microscope (Lambert Instruments, Netherlands) with modulated light source under the frequency domain. The data were stored as .fli files, and the $\tau$ (phase) for different structures (villi, muscle, crypts, and RBC) was determined by selecting multiple $(n=20)$ region of interests using fluorescein as the reference $(\tau=4 \mathrm{~ns})$. The data were then exported as excel files.

4.5. Imagery Analysis. All imagery data (.zvi file format) were analyzed using FIJI software after proper scaling. To compare the emission intensities between murine and porcine samples, the mean gray values were obtained (analyze $\rightarrow$ set measurements $\rightarrow$ select mean gray value and integrated density $\rightarrow$ measure) from 20 images $(n=20)$ and data were stored as .csv file for further statistical analysis. An arrow is drawn and then the distribution pattern was generated by using analyze $\rightarrow$ plot profile tool and numerical data were saved as excel sheet. For BoneJ analysis, cropped images of villous lamina propria were first binarized (process $\rightarrow$ binary $\rightarrow$ make binary) followed by skeletonizing and further purification/cleaning of data (plugins $\rightarrow$ BoneJ $\rightarrow$ skeletonise 3D $\rightarrow$ purify). The obtained data were then analyzed by analyze skeleton tool by selecting prune cycle method: none (plugins $\rightarrow$ BoneJ $\rightarrow$ analyze skeleton). The numerical data on mean branch length along with color-coded images for branching were obtained. The connective density $\left(\mu \mathrm{m}^{-3}\right)$ was determined using the connectivity tool (plugins $\rightarrow$ BoneJ $\rightarrow$ connectivity). The trabecular analysis on villous lamina propria was done using the thickness tool (plugins $\rightarrow$ BoneJ $\rightarrow$ thickness) to obtain data on trabecular thickness with SD. All data were exported as excel files for further analysis.

4.6. Statistics. The data $(n=20)$ were analyzed using OriginPro 2015 (OriginLab) software and plotted as mean \pm SD. Data points significantly different $(p<0.05)$ to control tissue (while assessing toxicity caused by QDs) or to murine RBCs (while comparing emission intensities between various murine and porcine villi, crypts, RBCs, and muscles) were marked with an asterisk $(*)$.

\section{ASSOCIATED CONTENT}

\section{Supporting Information}

The Supporting Information is available free of charge on the ACS Publications website at DOI: 10.1021/acsomega.8b00678.

Protocol for H\&E staining of tissue slides and excitation and emission spectra of CdSe QDs dispersed in toluene (PDF)

3D rendering of porcine jejunal villi from $z$-stack imaging based on tissue autofluorescence (AVI)

Isosurface view of murine villous lamina propria created by BoneJ plug-in of FIJI (AVI)

3D rendering of trabecular thickness of lamina propria in villi from murine jejunal tissue using FIJI software (AVI)

\section{AUTHOR INFORMATION}

\section{Corresponding Author}

*E-mail: sourav.bhattacharjee@ucd.ie. Phone: +353 1716 6271.

\section{ORCID}

Sourav Bhattacharjee: 0000-0002-6528-6877

\section{Notes}

The authors declare no competing financial interest.

\section{ACKNOWLEDGMENTS}

The authors would like to thank Philip Crilly (Kingston University, UK) and Prof. Torres Sweeney (UCD School of Veterinary Medicine) for support. S.B. would like to thank Dr. Tauseef Ahmad, Dr. Jason Beirne, and Patsy Kearns (UCD) for technological assistance. This study was funded by UCD OBRSS (Output Based Research Support Scheme) and OIP (Overhead Investment Plan) programmes. 


\section{ABBREVIATIONS}

CdSe, cadmium selenide; DPX, mixture of distyrene plasticizer and xylene; FLIM, fluorescence lifetime imaging microscopy; $\mathrm{H} \& \mathrm{E}$, hematoxylin and eosin; LED, light emitting diode; nm, nanometer; QD, quantum dot; ROI, region of interest; RBC, red blood corpuscle; SD, standard deviation; UV, ultraviolet; $\mathrm{mL}$, milliliter; $\mu \mathrm{g}$, microgram

\section{REFERENCES}

(1) Appel, A. A.; Anastasio, M. A.; Larson, J. C.; Brey, E. M. Imaging challenges in biomaterials and tissue engineering. Biomaterials 2013, 34, 6615-6630.

(2) Robertson, D.; Savage, K.; Reis-Filho, J. S.; Isacke, C. M. Multiple immunofluorescence labelling of formalin-fixed paraffin-embedded (FFPE) tissue. BMC Cell Biol. 2008, 9, 13.

(3) Monici, M. Cell and tissue autofluorescence research and diagnostic applications. Biotechnol. Annu. Rev. 2005, 11, 227-256.

(4) Kretschmer, S.; Pieper, M.; Hüttmann, G.; Bölke, T.; Wollenberg, B.; Marsh, L. M.; Garn, H.; König, P. Autofluorescence multiphoton microscopy for visualization of tissue morphology and cellular dynamics in murine and human airways. Lab. Invest. 2016, 96, 918931.

(5) Zipfel, W. R.; Williams, R. M.; Christie, R.; Nikitin, A. Y.; Hyman, B. T.; Webb, W. W. Live tissue intrinsic emission microscopy using multiphoton-excited native fluorescence and second harmonic generation. Proc. Natl. Acad. Sci. U.S.A. 2003, 100, 7075-7080.

(6) Croce, A. C.; Bottiroli, G. Autofluorescence spectroscopy and imaging: a tool for biomedical research and diagnosis. Eur. J. Histochem. 2014, 58, 2461.

(7) Farah, C. S.; McIntosh, L.; Georgiou, A.; McCullough, M. J. Efficacy of tissue autofluorescence imaging (velscope) in the visualization of oral mucosal lesions. Head Neck 2012, 34, 856-862.

(8) Singh, S. P.; Fält, P.; Barman, I.; Koistinen, A.; Dasari, R. R.; Kullaa, A. M. Objective identification of dental abnormalities with multispectral fluorescence imaging. J. Biophotonics 2017, 10, 12791286.

(9) Valdez, T. A.; Pandey, R.; Spegazzini, N.; Longo, K.; Roehm, C.; Dasari, R. R.; Barman, I. Multiwavelength fluorescence otoscope for video-rate chemical imaging of middle ear pathology. Anal. Chem. 2014, 86, 10454-10460.

(10) Auwärter, W.; Écija, D.; Klappenberger, F.; Barth, J. V. Porphyrins at interfaces. Nat. Chem. 2015, 7, 105-120.

(11) Monsel, A.; Lécart, S.; Roquilly, A.; Broquet, A.; Jacqueline, C.; Mirault, T.; Troude, T.; Fontaine-Aupart, M.-P.; Asehnoune, K. Analysis of autofluorescence in polymorphonuclear neutrophils: A new tool for early infection diagnosis. PLoS One 2014, 9, No. e92564.

(12) Hardman, R. A toxicologic review of quantum dots: Toxicity depends on physicochemical and environmental factors. Environ. Health Perspect. 2006, 114, 165-172.

(13) Lovrić, J.; Bazzi, H. S.; Cuie, Y.; Fortin, G. R. A.; Winnik, F. M.; Maysinger, D. Differences in subcellular distribution and toxicity of green and red emitting CdTe quantum dots. J. Mol. Med. 2005, 83, 377-385.

(14) Bottrill, M.; Green, M. Some aspects of quantum dot toxicity. Chem. Commun. 2011, 47, 7039-7050.

(15) Bhattacharjee, S.; Rietjens, I. M. C. M.; Singh, M. P.; Atkins, T. M.; Purkait, T. K.; Xu, Z.; Regli, S.; Shukaliak, A.; Clark, R. J.; Mitchell, B. S.; Alink, G. M.; Marcelis, A. T. M.; Fink, M. J.; Veinot, J. G. C.; Kauzlarich, S. M.; Zuilhof, H. Cytotoxicity of surface-functionalized silicon and germanium nanoparticles: the dominant role of surface charges. Nanoscale 2013, 5, 4870-4883.

(16) Bhattacharjee, S.; de Haan, L. H. J.; Evers, N. M.; Jiang, X.; Marcelis, A. T. M.; Zuilhof, H.; Rietjens, I. M. C.M.; Alink, G. M. Role of surface charge and oxidative stress in cytotoxicity of organic monolayer-coated silicon nanoparticles towards macrophage NR8383 cells. Part. Fibre Toxicol. 2010, 7, 25.

(17) Becker, W. Fluorescence lifetime imaging - techniques and applications. J. Microsc. 2012, 247, 119-136.
(18) Sollid, L. M. Coeliac disease: dissecting a complex inflammatory disorder. Nat. Rev. Immunol. 2002, 2, 647-655.

(19) Desnues, B.; Ihrig, M.; Raoult, D.; Mege, J.-L. Whipple's disease: a macrophage disease. Clin. Vaccine Immunol. 2006, 13, 170-178.

(20) Elliott, T. R.; Rayment, N. B.; Hudspith, B. N.; Hands, R. E.; Taylor, K.; Parkes, G. C.; Prescott, N. J.; Petrovska, L.; HermonTaylor, J.; Brostoff, J.; Boussioutas, A.; Mathew, C. G.; Bustin, S. A.; Sanderson, J. D. Lamina propria macrophage phenotypes in relation to Escherichia coli in Crohn's disease. BMC Gastroenterol. 2015, 15, 75. 